\title{
First incoherent scatter radar observations of ionospheric heating on the second electron gyro-harmonic
}

\author{
M.J. Kosch ${ }^{\text {a,* }}$, B. Gustavsson ${ }^{\text {b }}$, C. Heinselman ${ }^{c}$, T. Pedersen ${ }^{\text {d }}$, M.T. Rietveld ${ }^{\text {e }}$, J. Spaleta ${ }^{\text {f }}$, \\ A. Wong ${ }^{g}$, W. Wang ${ }^{g}$, C. Mutiso ${ }^{h}$, B. Bristow ${ }^{f}$, J. Hughes $^{h}$ \\ a Communication Systems, Lancaster University, Lancaster LA1 4WA, UK \\ b Department of Physics and Technology, University of Tromsø, N-9037 Tromsø, Norway \\ c Stanford Research Institute International, Menlo Park, CA 94025, USA \\ d Space Vehicles Directorate, Air Force Research Laboratory, Hanscom AFB, MA 01731, USA \\ e EISCAT Scientific Association, Ramfjordmoen, Ramfjordbotn N-9027, Norway \\ ${ }^{\mathrm{f}}$ Geophysical Institute, University of Alaska, Fairbanks, AK 99775, USA \\ ${ }^{g}$ HIPAS Observatory, Two Rivers, AK 99716, USA \\ ${ }^{\text {h }}$ Physical Science Dept., Embry-Riddle Aeronautical University, FL 32114, USA
}

\section{A R T I C L E I N F O}

Article history:

Received 20 March 2009

Received in revised form 29 July 2009

Accepted 22 August 2009

Available online 2 September 2009

Keywords:

Auroral ionosphere

Active experiments

\begin{abstract}
A B S T R A C T
We report first results from a unique experiment performed at the HIPAS ionospheric modification facility in conjunction with the Poker Flat incoherent scatter radar in Alaska. High-power radio waves at $2.85 \mathrm{MHz}$, which corresponds to the second electron gyro-harmonic at $\sim 245 \mathrm{~km}$ altitude, were transmitted into the nighttime ionosphere. Clear evidence of F-region ionospheric electron temperature enhancements were found, for the first time at this pump frequency, maximizing when the pump frequency is close to the second gyro-harmonic and double resonance. This is consistent with previous pump-enhanced artificial optical observations. We estimate the plasma heating efficiency to be approximately double that for higher pump frequencies.
\end{abstract}

(c) 2009 Elsevier Ltd. All rights reserved.

\section{Introduction}

Ionospheric modification resulting from high frequency (HF) high-power radio waves has found many applications in plasma physics (Leyser and Wong, 2009). Artificial optical emissions resulting from pump waves with O-mode polarization, are now routinely observed in the F-region at high latitudes since the original discovery in 1999 (Brändström et al., 1999). The pump waves interact with the ionospheric plasma, causing instabilities, which accelerate electrons up to $60 \mathrm{eV}$ or more (Gustavsson et al., 2005). These electrons collide with the atmospheric $O$ atoms and $\mathrm{N}_{2}$ molecules to produce aurora in the same way natural electron impact from precipitation does. Most of the important artificially stimulated auroral optical emissions have now been observed (Gustavsson and Eliasson, 2008) with the brightest optical emissions at O1D $630 \mathrm{~nm}$ and $\mathrm{O} 1 \mathrm{~S} 557.7 \mathrm{~nm}$. A review of $\mathrm{HF}$ pump-induced artificial optical phenomena is presented by Kosch et al. (2007a). In addition, the plasma is heated with electron temperatures up to $3500 \mathrm{~K}$ (Leyser et al., 2000), which can result in ion upwelling, and ion temperatures up to a few hundred $\mathrm{K}$

\footnotetext{
* Corresponding author.

E-mail address: m.kosch@lancaster.ac.uk (M.J. Kosch).
}

(Rietveld et al., 2003, 2004). However, plasma temperature enhancements are suppressed when pumping on a gyro-harmonic frequency (Robinson et al., 1996; Gustavsson et al., 2006) higher than the second electron gyro-harmonic. To date, the plasma temperature for pump frequencies near the second gyro-harmonic have not been reported, which is the objective of this manuscript.

Both the pump-induced optical emissions (Kosch et al., 2000; Pedersen and Carlson, 2001) and electron temperature enhancements (Rietveld et al., 2003, 2004) maximize sharply parallel to the local magnetic field line direction. Pedersen et al. (2003) found the full-width-at-half-maximum of the optical emissions to be $7^{\circ}$. Rietveld et al. $(2003,2004)$ found the electron temperature enhancement could drop from 3200 to $1800 \mathrm{~K}$ for $6^{\circ}$ off the magnetic zenith despite having the same pump power. The socalled magnetic zenith effect can boost the optical emission intensity by up to an order of magnitude and is the result of pump beam self-focusing (Kosch et al., 2007c).

The EISCAT and HAARP ionospheric modification facilities, located in Norway and Alaska, respectively, are capable of stimulating a wide range of plasma instabilities, including parametric decay instabilities, upper-hybrid resonance and the thermal parametric instability. Kosch et al. (2002) showed that artificial optical emissions are closely associated with the upperhybrid resonance (UHR), which is stimulated typically a few 
kilometers below the HF reflection altitude $\left(f_{o}=f_{p}\right)$ according to $f_{o}^{2}=f_{U H R}{ }^{2}=f_{p}{ }^{2}=f_{G H}{ }^{2}$, where $f_{o}$ is the pump frequency, $f_{p}$ the local plasma frequency, and $f_{G H}$ the electron gyro-frequency. $f_{G H}$ is a function of magnetic field strength and therefore altitude in the ionosphere. Upper-hybrid resonance has a symbiotic relationship with meter-scale magnetic field-aligned plasma density irregularities, called striations, which are observed as enhanced backscatter by the SuperDARN radars (Kosch et al., 2002; and references therein). The artificial optical emissions and striations are both suppressed when pumping on a gyro-harmonic frequency (Kosch et al., 2002; Gustavsson et al., 2006) higher than the second electron gyro-harmonic. However, Kosch et al. (2007b) found no minimum in SuperDARN radar backscatter for pumping on the second gyro-harmonic. In addition, Kosch et al. (2005) found an optical maximum for pump frequencies just above second electron gyro-harmonic (about $7 \mathrm{kHz}$ ), i.e. the opposite compared to higher gyro-harmonics.

Haslett and Megill (1974) first reported unusually large optical enhancements when pumping the ionosphere at twice the electron gyro-frequency. The pump-induced optical emissions on the second gyro-harmonic have similar intensities compared to higher pump frequencies, which used an order of magnitude more HF power (Kosch et al., 2007a). Pedersen et al. (2008) showed that for modest effective radiated power (ERP) less than $40 \mathrm{MW}$, the induced optical intensity was linearly related to ERP. In addition, they show data from a HAARP experiment (their Fig. 1) of optical enhancements from ionospheric pumping at 3.3 and $2.84 \mathrm{MHz}$ with 42.4 and $32.1 \mathrm{MW}$ ERP, respectively, within the same half hour period. Although not directly addressed by the authors, it is easily shown that $630 \mathrm{~nm}$ optical emission intensity at $2.84 \mathrm{MHz}$, i.e. on the second gyro-harmonic, is approximately double that at 3.3 $\mathrm{MHz}$, i.e. away from the second gyro-harmonic, including linear compensation for the change in ERP. This result has been subsequently confirmed at HAARP using 2.85 and $3.15 \mathrm{MHz}$ pump frequencies (Pedersen, unpublished manuscript).

For O-mode pumping near the second electron gyro-harmonic frequency, Kosch et al. (2007b) have determined which groupings of mechanisms are acting. For pump frequencies below the second gyro-harmonic in an over-dense ionosphere, two parametric decay instabilities are active. First, the pump wave can decay into an ion-acoustic and electron Langmuir waves. These can be detected as strong enhancements in the ion- and plasma-lines of incoherent scatter radars (Rietveld et al., 2000). Unfortunately, such enhancements also prevent plasma temperature analysis of the radar spectra. Second, the pump wave can decay into an electron-Bernstein and lower-hybrid waves. The latter can be detected by SuperDARN radars as lower-hybrid caviton collapse. For pump frequencies above the second electron gyro-harmonic in an over-dense ionosphere, two parametric decay instabilities and the thermal parametric instability are active. First, the pump wave can decay into an ion-acoustic and electron Langmuir waves, as before. Second, the pump wave can decay into an upper-hybrid and lower-hybrid waves. The former can be detected by SuperDARN radars as striations. Third, the thermal parametric instability results in upper-hybrid waves and striations, which can be detected by SuperDARN radars, as before. Finally, for pump frequencies above the second gyro-harmonic in an under-dense ionosphere where $f_{o} \leq f o F 2+0.5 \mathrm{MHz}$ (Pedersen et al., 2003), one parametric decay instability and the thermal parametric instability are active. First, the pump wave can decay into an upperhybrid and lower-hybrid waves, as before. Second, the thermal parametric instability results in upper-hybrid waves and striations, also as before. Both mechanisms can be detected by SuperDARN radars as striations. Once upper-hybrid resonance is no longer possible in the under-dense ionosphere all optical emissions cease.
To measure the plasma temperature resulting from $\mathrm{HF}$ pumping of the ionosphere, an incoherent scatter radar is necessary. Currently, the only available experimental combination for the second electron gyro-harmonic frequency is the HIPAS ionospheric modification facility, operating on $2.85 \mathrm{MHz}$, and the newly inaugurated Poker Flat Advanced Modular Incoherent Scatter Radar (AMISR), called PFISR, both located in Alaska. The EISCAT facility does have two co-located incoherent scatter radars, but the minimum pump frequency only reaches down to the third electron gyro-harmonic ( $\sim 4 \mathrm{MHz})$. For a few years (1980-1985), EISCAT did have second gyro-harmonic pumping capability $(2.75 \mathrm{MHz})$, but a thorough survey of the archive data shows that no suitable experiment was ever performed. Unfortunately, the HAARP ionospheric modification facility does not have an incoherent scatter radar available. Here we report the first electron temperature observations resulting from ionospheric pumping on the second electron gyro-harmonic.

\section{Results and discussion}

On 19 March 2007 the HIPAS facility in Alaska $\left(64.87^{\circ} \mathrm{N}\right.$, $146.84^{\circ} \mathrm{W}$ ) was operated in a 6 -min cycle of 3 phases: 2 min on pulsing, 2 min continuous wave, and 2 min off. Operations started at 04:24 UT and ended at 06:58 UT. The HF pump beam was pointed parallel to the magnetic field line direction $\left(77^{\circ}\right.$ elevation, $204^{\circ}$ azimuth) with O-mode polarization for continuous wave pumping, transmitting at $2.85 \mathrm{MHz}$ with $\sim 32 \mathrm{MW}$ ERP in the centre of the beam, and a full beam width of $23.3^{\circ} .2 .85 \mathrm{MHz}$ corresponds to the second electron gyro-harmonic at $\sim 245 \mathrm{~km}$ altitude over HIPAS. Pointing the beam into the magnetic zenith is done specifically because it greatly enhances the optical emissions (Kosch et al., 2000; Pedersen and Carlson, 2001) and electron temperature enhancements (Rietveld et al., 2003, 2004). During the pulsing phase, the beam pointing was $77^{\circ}$ elevation and $246^{\circ}$ azimuth. From 04:24 to 06:08 UT pulsing consisted of $50 \mathrm{~ms}$ on every $4 \mathrm{~s}$, and from 06:12 to 06:58 UT pulsing consisted of $50 \mathrm{~ms}$ on every second. The pulse modes did not produce any detectable effects for our purpose. Here we focus on the continuous wave transmissions only.

The College ionosonde $\left(64.9^{\circ} \mathrm{N}, 148.0^{\circ} \mathrm{W}\right)$ is used to characterize the ionosphere. A frequency sweep, starting at $0.2 \mathrm{MHz}$, was done every $2 \mathrm{~min}$. Although College is $\sim 55 \mathrm{~km}$ west of HIPAS, the fields of view overlap in the F-layer. Geomagnetic activity was very low on $19 / 03 / 2007$ with $\mathrm{Kp}<=1^{-}$in the $12 \mathrm{~h}$ up to and including the experiment. Fig. 1 shows HF reflection (solid curve), upper-hybrid resonance (dashed curve), and second electron the gyro-harmonic (dot-dashed curve) altitudes, all for $2.85 \mathrm{MHz}$. The ionosphere is over-dense until $\sim 05: 40 \mathrm{UT}$, thereafter underdense. Double resonance, i.e. where the upper-hybrid frequency also equals the gyro-harmonic frequency, occurs for the interval $\sim 05: 58-06: 06$ UT. Upper-hybrid resonance stops at $\sim 06: 08$ UT. Fig. 1 shows that HF reflection and upper-hybrid resonance both cease shortly after intersecting with the second gyro-harmonic altitude. Hence, for almost the entire experiment, the HIPAS pump frequency corresponded to pumping below the second gyroharmonic. Unfortunately, the mechanisms acting in this frequency regime produce low intensity optical emissions compared to above the second gyro-harmonic (Kosch et al., 2007b, c).

The first useful optical recordings started at $\sim 05: 20$ UT due to sunset, using $630 \mathrm{~nm}$ ( $8 \mathrm{~s}$ exposures every $10 \mathrm{~s}$ ) and $557.7 \mathrm{~nm}$ (7 s exposure every $10 \mathrm{~s}$ ). The sky was completely clear of clouds. Fig. 2 shows un-calibrated background-subtracted images of the pump-induced artificial optical emissions at $630 \mathrm{~nm}$ taken from HIPAS at the end of each continuous wave pump cycle. The intensity and size of the optical emission region remains fairly 


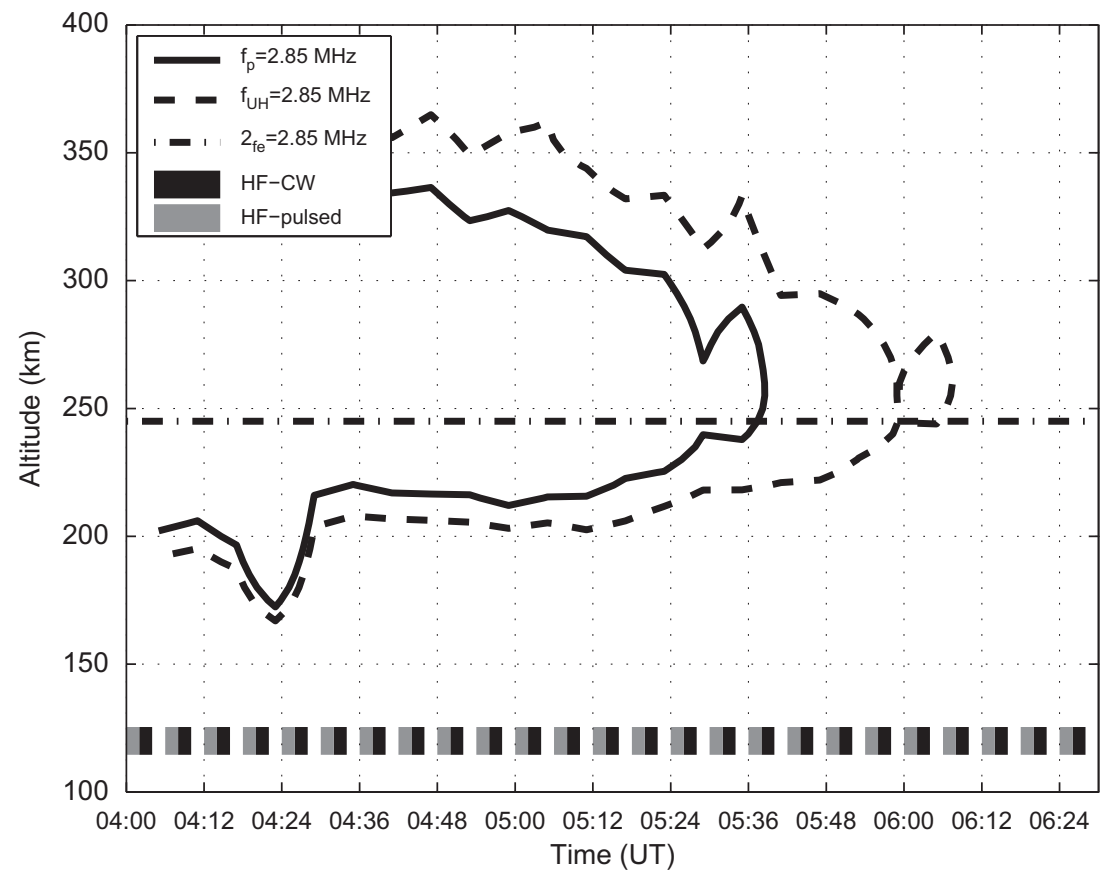

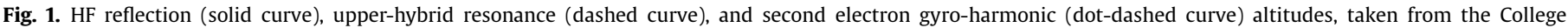
ionosonde for $2.85 \mathrm{MHz}$. HIPAS was operated in pulsed (grey panels) and continuous wave (black panels) modes with other periods having no transmissions.

HIPAS 19 March 2007

05:28 UT

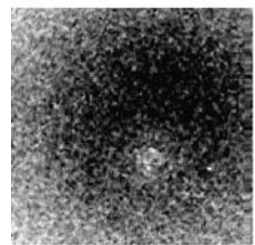

05:52 UT

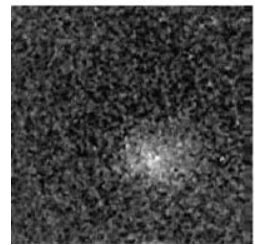

05:34 UT

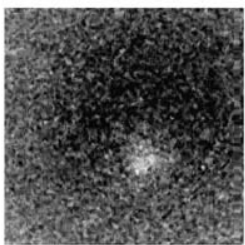

05:58 UT

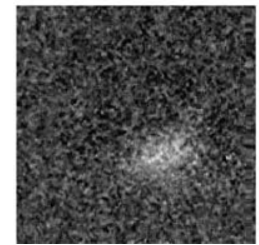

05:40 UT

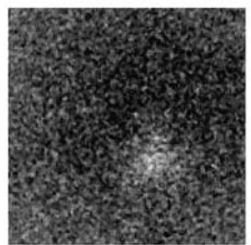

06:04 UT

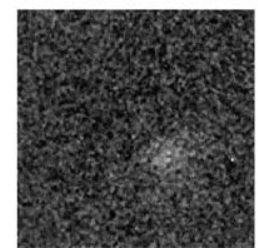

05:46 UT

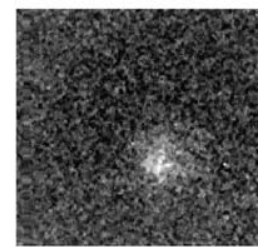

06:10 UT

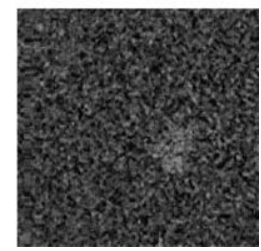

Fig. 2. Un-calibrated background-subtracted images of the pump-induced artificial optical emissions at $630 \mathrm{~nm}$ taken from HIPAS at the end of each continuous wave pump pulse. The field of view is $\sim 45^{\circ}$. North and west are to the top and right of the images, respectively. The "halo" apparent in the earlier images is an artifact of background subtraction caused by declining twilight.

constant throughout the experiment, fading towards the end as the pump frequency exceeds the ionospheric critical frequency. A detailed description of the optical results from this experiment can be found in Gustavsson et al. (2008). In addition, Mutiso et al. (2008) reported the first observation of the $\mathrm{O}^{+} 732 \mathrm{~nm}$ optical emission from this campaign. Here we focus on the radar results.

The Poker Flat Incoherent Scatter Radar (PFISR, $65.13^{\circ} \mathrm{N}$, $147.47^{\circ} \mathrm{W}$ ) observed the ionosphere with four beams, beam 1 pointing into the local magnetic zenith, and beams 2, 3 and 4 intersecting near the local zenith over HIPAS at approximately 300,240 and $200 \mathrm{~km}$, respectively. The experimental geometry is shown in Fig. 3. From the pre-programmed beams available, beams 2,3 and 4 corresponding to azimuth angles of $145.34^{\circ}$, $135.13^{\circ}, 129.24^{\circ}$, and elevation angles of $82.12^{\circ}, 79.58^{\circ}, 76.82^{\circ}$, respectively, were closest to the desired positions. The radar was swept through all four beams, alternating long pulse with $35 \mathrm{~km}$ range resolution and alternating code with $4.5 \mathrm{~km}$ range resolution, and a total integration time of $6.144 \mathrm{~s}$. The PFISR is located $29.3 \mathrm{~km}$ east and $28.7 \mathrm{~km}$ north of HIPAS, and the antenna face is tilted northward. Hence, unfortunately, it is impossible for PFISR to intersect with the magnetic field line passing through HIPAS. This is a significant geometric limitation of the experiment as the PFISR beams can only intersect the HIPAS beam near its $-1 \mathrm{~dB}$ power contour for the chosen altitudes when HIPAS is pointed into its local magnetic zenith, as was the case on 19/03/ 2007. The geometry corresponds to PFISR pointing $\sim 7^{\circ}$ away from HIPAS's magnetic zenith, i.e. on the edge of the region of maximum optical intensity and electron temperature enhancement. The geometry effectively precludes PFISR observations of any advantage from the self-focusing effect 
within the HF pump beam (Kosch et al., 2007c), which is associated with a significant gain in pump power density. If anything, PFISR is pointing into a region of diminishing pump power as self-focusing progresses. PFISR construction was not completed at the time of the campaign, and the alternating code data were very noisy, so we focus on the long-pulse data only.

Fig. 4 shows the PFISR ion-line long-pulse data, post-integrated to $30.72 \mathrm{~s}$, for each of the four beams used. The pump-on periods which show enhanced plasma density, especially apparent in the PFISR field-aligned beam 1 around $200-250 \mathrm{~km}$ prior to 05:30 UT, correspond to the ion-line enhancement phenomenon. These are observed at EISCAT (Ashrafi et al., 2006, 2007) and HAARP (Kosch et al., 2007b; Oyama et al., 2007) and are associated with pumpinduced Langmuir turbulence, not real plasma density

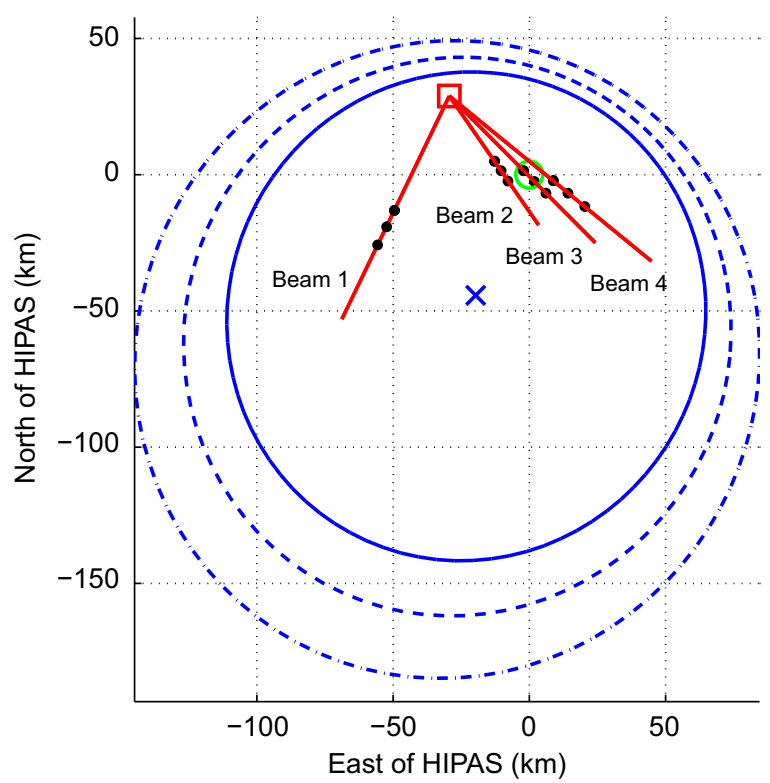

Fig. 3. A sketch of the experimental geometry. All distances are relative to the HIPAS site (green circle). PFISR is located at the red square. The direction of the 4 radar beams are shown as red lines, with black markers at 210, 240 and $274 \mathrm{~km}$. The blue ovals denote the HIPAS beam $-3 \mathrm{~dB}$ contour for $210 \mathrm{~km}$ (solid), $240 \mathrm{~km}$ (dashed) and $274 \mathrm{~km}$ (dash-dotted) altitude. The blue cross shows the magnetic field line position for $210 \mathrm{~km}$ altitude.(For interpretation of the references to color in this figure legend, the reader is referred to the web version of this article.) enhancements. Data prior to 05 UT are not shown because it is contaminated by the pump-induced ion-line enhancement. From $\sim 05: 30$ UT onwards, the ion-line overshoot increases in altitude, disappearing after 05:41 UT. This consistent with the ionosonde data (see Fig. 1), which shows a similar increase in altitude of the HF reflection altitude, with reflection ceasing around 05:40 UT. It is the pump-driven parametric decay instability near the HF reflection altitude, where the pump wave decays into an electron Langmuir wave and an ion-acoustic wave, which manifests itself as the ion-line enhancement (Kosch et al., 2007b). This is expected to cease when HF reflection ceases, as shown in Figs. 1 and 4.

Fig. 4 also shows some electron temperature enhancements, although the plot cannot be taken completely at face value because the PFISR spectra affected by the ion-line enhancement may not be correctly analysed. However, there are altitudes and times without the ion-line enhancement where modest electron temperature enhancements are apparent. Electron temperature enhancements seen after 05:48 UT in beam 1, 05:36 UT in beams 2 and 3, and 05:30 UT in beam 4 are not contaminated and may be taken at their face value. No ion temperature enhancements were found (not shown). The relatively modest effective radiated power of the HIPAS facility coupled to the relatively short pump periods (2 min) mitigate against ion temperature enhancements. Such observations at EISCAT are associated with high effective radiated powers ( $\sim 200 \mathrm{MW}$ ) and pump periods up to $8 \mathrm{~min}$ (Rietveld et al., 2003, 2004).

Fig. 5 shows the calibrated intensity at $630 \mathrm{~nm}$ of the pumpinduced artificial optical emissions above twilight background. The continuous wave pump-on periods clearly show O1D enhancements up to 100 Rayleighs above background. This is consistent with the $4 \mathrm{R} / \mathrm{MW}$ calibration $(4 * 32=128 \mathrm{R})$ found by Pedersen et al. (2008). The optical background is variable due to faint natural airglow. The optical enhancements remain strong up to $\sim 06$ UT whilst upper-hybrid resonance remains possible (see Figs. 1 and 2). Once upper-hybrid resonance ceases, the optical enhancements decline but are still evident up to 06:22 UT, sustained by patches of ionosphere with enhanced plasma density.

Fig. 5 also shows the electron temperature taken from the three PFISR beams intersecting the HIPAS pump beam at three different altitudes, post-integrated to $30.72 \mathrm{~s}$. The data gaps are from HF-contaminated ion-line enhanced spectra that could not be analysed, which have been removed. Electron temperature enhancements up to $\sim 500 \mathrm{~K}$ above background are evident,

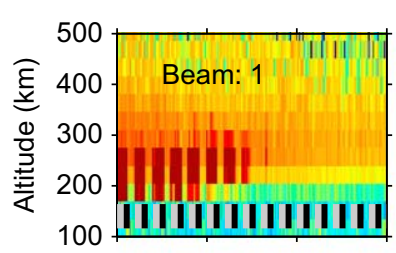

$\log 10$ Electron density $\left(\mathrm{m}^{-3}\right)$
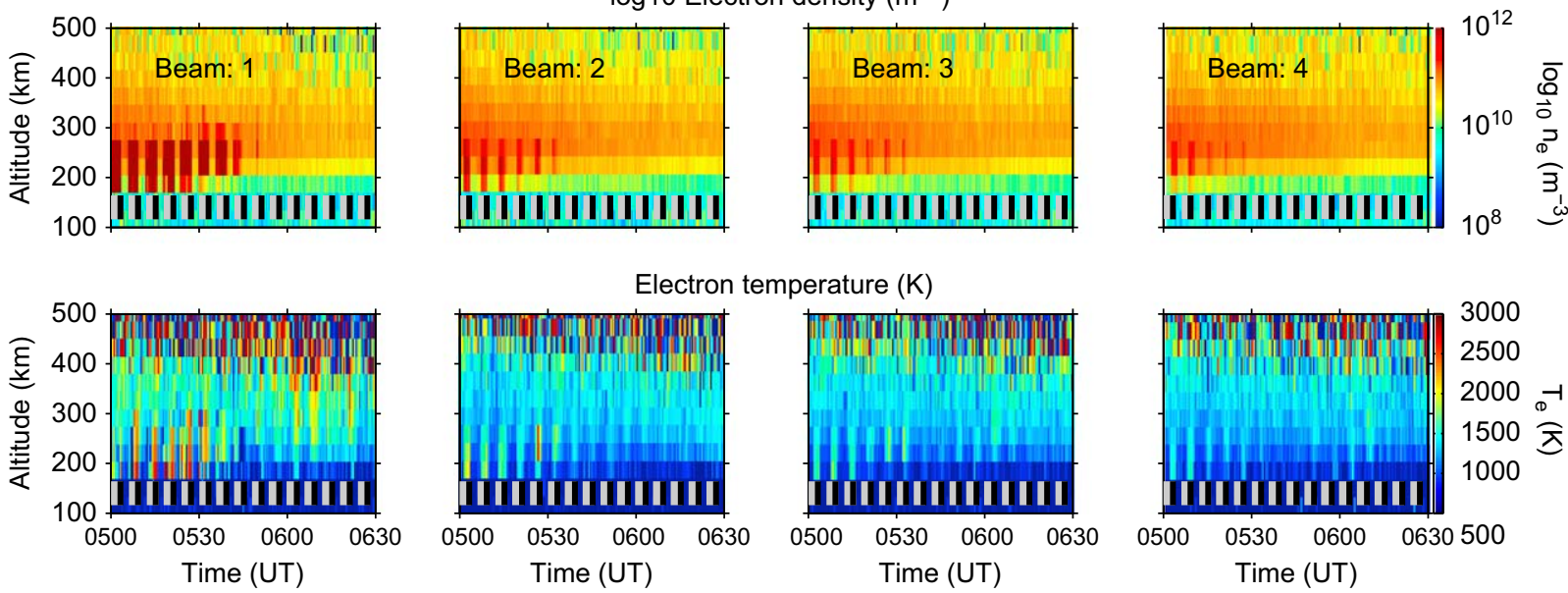

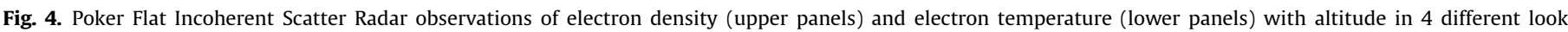

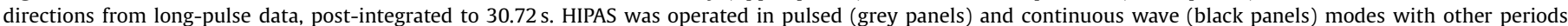
having no transmissions. 


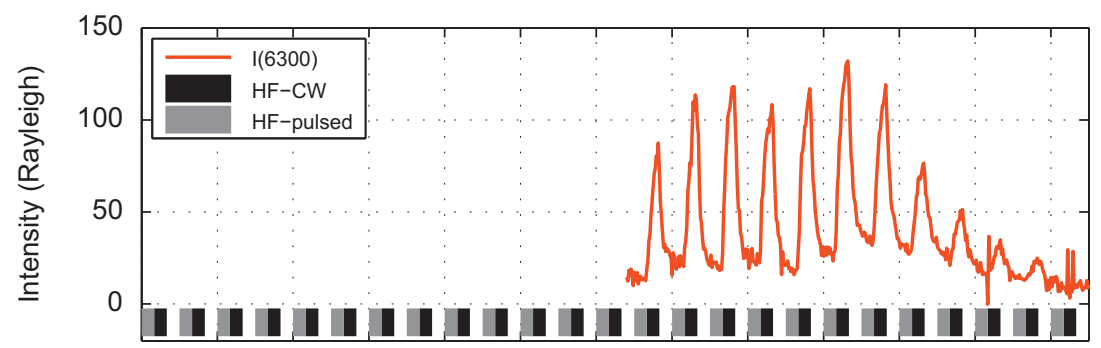

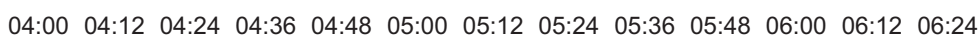

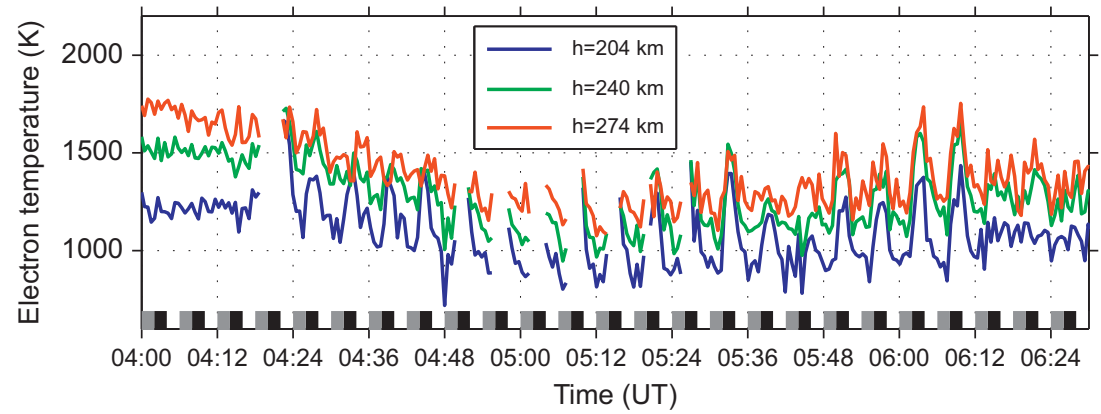

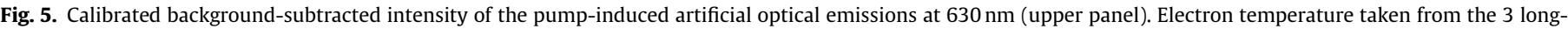

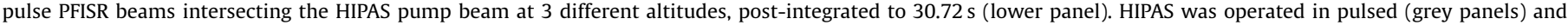
continuous wave (black panels) modes with other periods having no transmissions.

starting right from the first HIPAS continuous wave pulse at 04:26 UT. Just as is the case for the optical emissions, electron temperature enhancements continue up to 06:22 UT, hence there is a clear association with the optical emissions as well as upperhybrid resonance. This is consistent with the findings at EISCAT for higher pump frequencies (Kosch et al., 2002; Gustavsson et al., 2006). It is also noted that the maximum electron temperature enhancement occurs for the HIPAS continuous wave pulses at 06:02 and 06:08 UT when the ionosonde data (see Fig. 1) shows that double resonance is active. This is consistent with the optical intensity maximum observed at HAARP (Kosch et al., 2005, 2007b), although it is not obvious in the HIPAS optical data. In addition, there is another maximum for the pulse at 05:32 UT, corresponding to the time when the pump frequency was close to the ionospheric critical frequency. In this situation, the plasma density vertical gradient is small, thereby giving a long interaction path for the pump wave. Hence, it is expected that coupling between the pump wave and the plasma would maximize.

The electron temperature enhancements are relatively modest compared those observed at EISCAT, which can be up to $3500 \mathrm{~K}$ (Leyser et al., 2000; Rietveld et al., 2003, 2004). However, given that the PFISR beams do not intersect the HIPAS magnetic field line, i.e. not at the point of maximum power in the centre of the pump beam, as described above, it is probable that the peak electron temperature in the centre of the HIPAS pump beam is up to $\sim 900 \mathrm{~K}$, based on EISCAT beam-swinging experiments (Rietveld et al., 2003, 2004). Simple geometric considerations (see Fig. 3) and modeling of the HIPAS beam show that the PFISR beams were probing plasma illuminated by $\sim 25 \mathrm{MW}$ effective radiated power, excluding any reduction due to pump beam self-focusing, which is approximately an order of magnitude less than that typically used at EISCAT for similar experiments. HIPAS achieved $500 / 25=20 \mathrm{~K} / \mathrm{MW}$ at $2.85 \mathrm{MHz}$, possibly more if pump beam selffocusing is taken into account, whereas EISCAT achieved 1400/ $200=7 \mathrm{~K} / \mathrm{MW}$ at $4.544 \mathrm{MHz}$ for a similar geometry, i.e. $6^{\circ}$ away from the magnetic field line direction. Unfortunately, the pump power effect on electron temperature enhancements has never been quantified for nighttime experiments. Assuming linearity, and adjusting for the difference in plasma density associated with the pump frequency at HIPAS $(2.85 \mathrm{MHz})$ and EISCAT $(4.544 \mathrm{MHz})$, the difference in ERP at HIPAS (25 MW) and EISCAT (200 MW), and the approximate height difference between the HIPAS $(220 \mathrm{~km})$ and EISCAT $(280 \mathrm{~km})$ experiments, the observed electron temperature difference between HIPAS (500 K) and EISCAT (1400 K) indicates that the plasma heating efficiency on the second gyro-harmonic is approximately double that for a higher pump frequency $\left(\left(2.85^{2} / 4.544^{2}\right) \times(500 / 1400) \times\left(280^{2} / 220^{2}\right) \times\right.$ $(200 / 25)=1.82)$ for the same pump beam geometry relative to the magnetic field line, which is consistent with the fact that the optical intensity produced on the second gyro-harmonic is greater than for higher pump frequencies of the same HF power (Pedersen et al., 2008). However, the experiment described here was almost entirely for pumping below the second gyro-harmonic (see Fig. 1). HAARP experiments show that the optical emissions can more than double for pumping above the second gyro-harmonic (Kosch et al., 2005, 2007b, c). Hence, it is expected that the electron temperature enhancement might be even greater, possibly by another factor of two, than those reported here for this frequency regime.

The Kodiak SuperDARN radar was operated in a special mode 03-06 UT, thereafter performing a standard scan of 16 beams every $2 \mathrm{~min}$ at one frequency. In the special mode, the radar was swept through frequencies close to $8,9,10,11$ and $12 \mathrm{MHz}$, and cycling through beams 1-3 only, with $1 \mathrm{~s}$ integration and $15 \mathrm{~km}$ range resolution, making a $15 \mathrm{~s}$ cycle. Frequency selection in each band was automatically adjusted to achieve minimum interference. Fig. 6 shows SuperDARN Kodiak radar backscatter power averaged over beams $1-3$ and frequencies $9.8-10.2 \mathrm{MHz}$ for the range gate over HIPAS. Backscatter enhancements of $10-15 \mathrm{~dB}$ occur during the continuous wave phase of each HIPAS cycle. The backscatter enhancement prior to $04: 24 \mathrm{UT}$ is due to HIPAS transmitter tune-up. It is clear that the backscatter does not go completely back to the initial background state, as seen prior to 04:10 UT, when the pump is turned off. This may be due to the relatively short pump-off period $(2 \mathrm{~min})$ and ionospheric preconditioning during the pump-on phases $(4 \mathrm{~min})$ and is consistent with HAARP observations (Kosch et al., 2005, 2007b). The Doppler shift (not shown) is less than $\pm 50 \mathrm{~m} / \mathrm{s}$ but not 


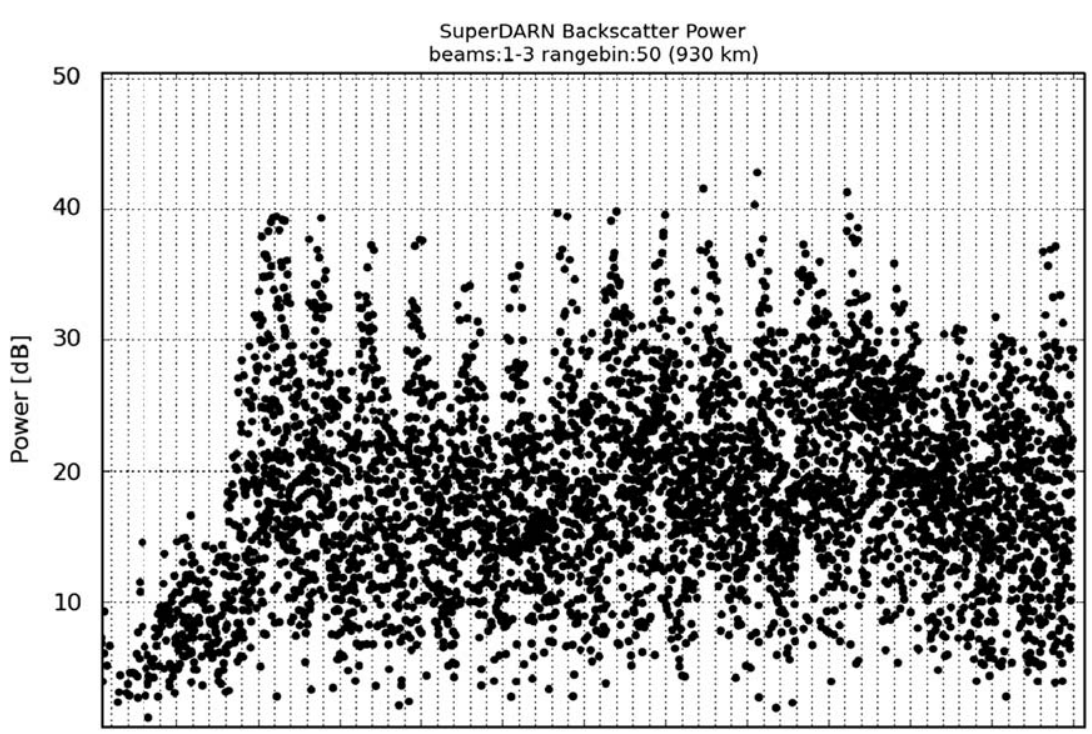

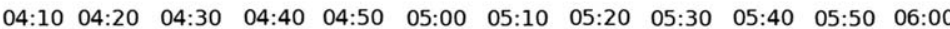

Time UTC

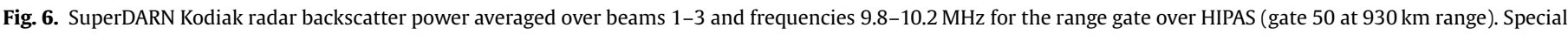
mode radar operations ceased at 06 UT before the experiment ended.

persistently equal to zero and therefore the backscatter is not due to ground scatter. The spectral width parameter (not shown) is less than $25 \mathrm{~m} / \mathrm{s}$, which indicates a relatively hard target consistent with ionospheric modification experiments. These are the first observations of HIPAS-induced SuperDARN radar backscatter modulation. These observations correspond to the parametric decay instabilities of Langmuir and electron-Bernstein waves being active, i.e. not upper-hybrid resonance, because most of the experiment occurred for pump frequencies below the second gyro-harmonic. Therefore, the Kodiak radar HIPAS-induced backscatter comes from lower-hybrid wave caviton collapse and not field-aligned striations, as described by Kosch et al. (2007b).

The observed electron temperature profiles give direct estimates of the pump-induced electron heating after integrating the one-dimensional energy equation:

$\frac{3}{2} n_{e} k_{B} \frac{\partial T_{e}(z, t)}{\partial t}=\frac{\partial}{\partial z}\left(K_{e}\left(T_{e}\right) \frac{\partial T_{e}(z, t)}{\partial z}\right)+Q_{H F}(z, t)-Q_{o}(z, t)-L_{e}\left(T_{e}\right)$

where $n_{e}$ and $T_{e}$ are the electron density and temperature, respectively, $k_{B}$ Boltzmann's constant, $K_{e}$ heat flux, $Q_{H F}$ pump energy input, $Q_{o}$ the heat input from other sources, $L_{e}$ the electron energy loss function, $t$ time, and $z$ altitude. The electron cooling is calculated with electron temperature observations from the last $90 \mathrm{~s}$ of the pump-on and pump-off periods, during which we consider $T_{e}$ to be at steady state. Taking the difference leads to:

$$
\begin{aligned}
\int_{z_{o}}^{z_{1}} Q_{H F} & =\int_{z_{o}}^{z_{1}} L_{e}\left(T_{e}^{H F}\right)-L_{e}\left(T_{e}^{O F F}\right) d z \\
& +\left|K_{e}\left(T_{e}^{O F F}\right) \frac{\partial T_{e}^{O F F}}{\partial z}\right|_{z_{o}}^{z_{1}}-\left|K_{e}\left(T_{e}^{H F}\right) \frac{\partial T_{e}^{H F}}{\partial z}\right|_{z_{o}}^{z_{1}}
\end{aligned}
$$

We integrate the electron energy loss function $\left(L_{e}\right)$ from 150 to $375 \mathrm{~km}$. The heat flux $\left(K_{e}\left(T_{e}\right)\left(\partial T_{e} / \partial z\right)\right)$ is small through the lower boundary, for both pump-on and pump-off conditions. Unfortunately, we cannot take into account the energy lost by heat flowing up to altitudes higher than $375 \mathrm{~km}$ because the PFISR data have low signal-to-noise ratio at high altitudes. However, this should be a relatively minor correction when considering that the electron temperature modulation at altitudes above $350 \mathrm{~km}$ is significantly reduced compared to $250 \mathrm{~km}$ (not shown). The processes taken into account for electron cooling are elastic collisions with ions and the major neutral species (e.g. Schunk and Nagy, 2004) with cooling rates from Rees and Roble (1975) and Stubbe and Varnum (1972), rotational and vibrational excitation of $\mathrm{N}_{2}$ and $\mathrm{O}_{2}$ with cooling rates from Pavlov (1998a, b), cooling due to excitation of the fine structure levels of $O$ (Pavlov and Berrington, 1999), and excitation of O1D with cooling rates calculated from the excitation rate of Mantas and Carlson (1991). By integrating the electron energy losses we get an estimate of the energy transferred from the pump wave to the ionospheric electrons.

The total pump-induced electron heating with altitude is shown in Fig. 7 upper panel, and integrated in altitude from 150 to $375 \mathrm{~km}$ is shown in Fig. 7 lower panel, for all four PFISR beams. There is significant modulation due to HF pumping, ranging from $6 \times 10^{12}$ to $6 \times 10^{13} \mathrm{eV} / \mathrm{m}^{2} / \mathrm{s}$. The data are also adversely affected by the ion-line enhancement phenomenon, especially in the earlier part of the experiment. The electron temperature is measured by PFISR near the $-1 \mathrm{~dB}$ contour of the HF pump beam. Taking this into account as well as the lower power density of HIPAS, the energy-transfer efficiency is comparable to that which Sergienko et al. (2000) used to model the electron temperature above EISCAT, and that which Robinson (1989) estimated was deposited to explain anomalous absorption observations.

By measuring the electron temperature profile at different cuts through the HF beam it is in principle possible to estimate the variation of the energy-transfer efficiency as a function of effective radiated power and angle relative to magnetic zenith. However, the PFISR beams cut the HIPAS beam at approximately similar contours of the antenna pattern and angles relative to the magnetic field, making it difficult to draw any conclusions about geometric variations in heating efficiency. Such work requires colocated ionospheric modification and incoherent scatter radar facilities.

\section{Conclusion}

The discovery at HAARP that bright pump-induced artificial optical emissions, which are due to accelerated electrons, occur 

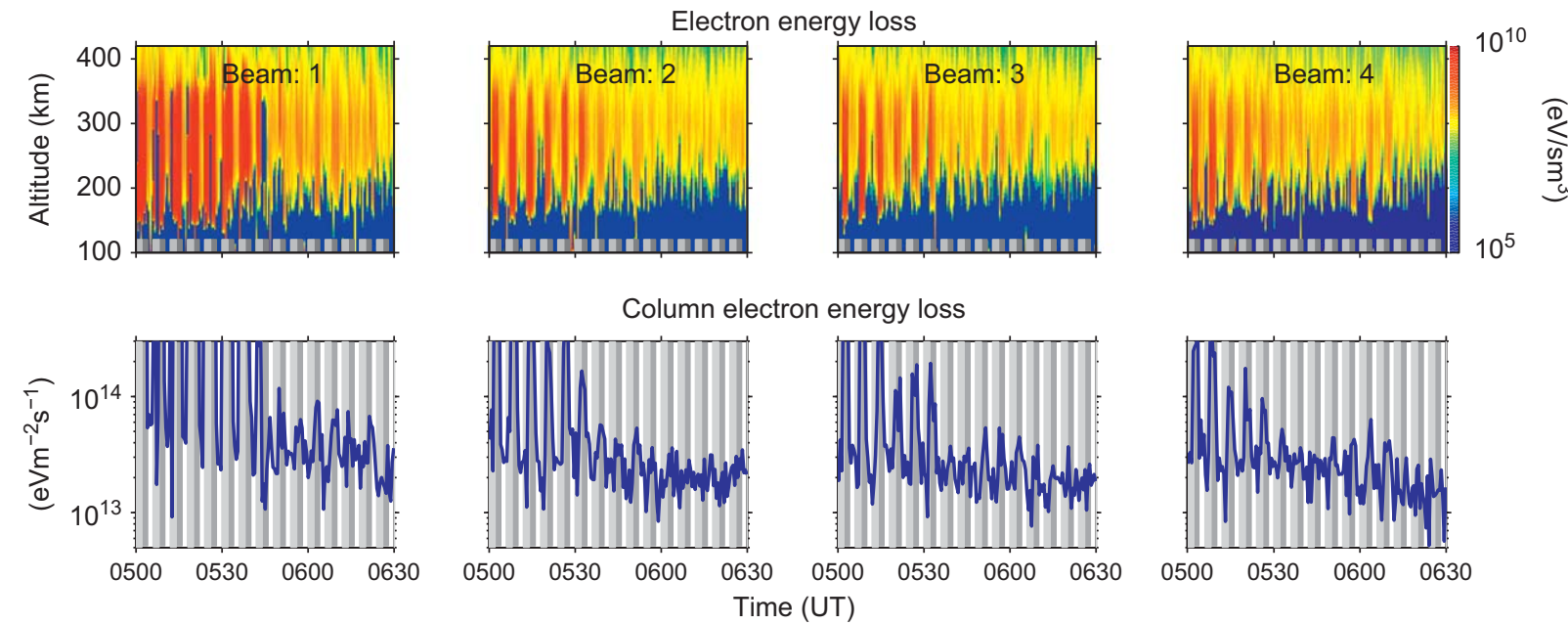

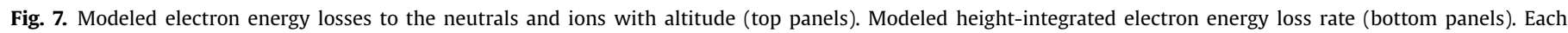

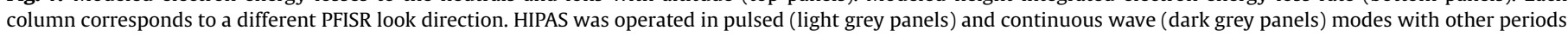
having no transmissions.

for pump frequencies a few kilohertz above the second electron gyro-harmonic frequency in the ionospheric F-layer clearly indicates that powerful wave-plasma resonances are taking place. Unfortunately, the HAARP facility does not have a co-located incoherent scatter radar to diagnose the plasma. The EISCAT ionospheric modification facility does have two co-located incoherent scatter radars, but the minimum pump frequency only reaches down to the third electron gyro-harmonic. Although the experiment geometry was unfavourable, the only available possibility was to use the HIPAS ionospheric modification facility in conjunction with the newly inaugurated PFISR in Alaska. This has clearly demonstrated the enhanced artificial optical emissions correspond to enhanced ionospheric electron temperatures, peaking for pump frequencies near the second electron gyroharmonic frequency and near double resonance. In addition, the efficiency of plasma heating is likely to be approximately double, or more, for pumping near the second gyro-harmonic compared to higher frequencies, based on the intensity of the pump-induced optical emissions. We also report the first SuperDARN radar observation of enhanced HF backscatter over HIPAS.

\section{Acknowledgements}

Funding for HIPAS operations is gratefully acknowledged from The International Foundation for Science, Health and Environment. We acknowledge the assistance of P. O'Connor in processing the optical images.

\section{References}

Ashrafi, M., Kosch, M.J., Honary, F., 2006. Heater-induced altitude descent of the EISCAT UHF ion-line overshoot: Observations and modeling. Adv. Space Res 38, 2645-2652.

Ashrafi, M., Kosch, M.J., Kaila, K., Honary, F., 2007. Spatio-temporal evolution of HFinduced artificial optical emissions, radar backscatter and Stimulated Electromagnetic Emissions at EISCAT. J. Geophys. Res. 112, A05314, doi:10.1029/ 2006JA011938.

Brändström, B.U.E., Leyser, T.B., Steen, A., Rietveld, M.T., Gustavsson, B., Aso, T., Ejiri M., 1999. Unambiguous evidence of HF pump-enhanced airglow at auroral latitudes. Geophys. Res. Lett. 26, 3561-3564.

Gustavsson, B., Eliasson, B., 2008. HF radio wave acceleration of ionospheric electrons: analysis of HF-induced optical enhancements. J. Geophys. Res. 113 doi:10.1029/2007JA012913.

Gustavsson, B., Sergienko, T., Kosch, M.J., Rietveld, M.T., Brändström, B.U.E., Leyser, T.B., Isham, B., Gallop, P., Aso, T., Ejiri, M., Grydeland, T., Steen, A., LaHoz, C.,
Kaila, $\mathrm{H}$, Jussila, J., Holma, $\mathrm{H}$, 2005. The electron distribution during HF pumping, a picture painted with all colours. Ann. Geophys. 23, 1747-1754.

Gustavsson, B., Leyser, T.B., Kosch, M.J., Rietveld, M.T., Steen, A., Brändström, B.U.E., Aso, T., 2006. Electron gyroharmonic effects in ionization and electron acceleration during HF pumping in the ionosphere. Phys. Rev. Lett. 97, 190052.

Gustavsson, B., Kosch, M., Wong, A., Pedersen, T., Heinselman, C., Mutiso, C., Bristow, B., Hughes, J., Nielsen, K., Wang, W., 2008. First estimates of altitude distribution of HF-pump enhanced emissions at 6300 and $5577 \mathrm{~A}$ : a comparison between observations and theory. Ann. Geophys. 26, 3999-4012.

Haslett, J.C., Megill, L.R., 1974. A model of the enhanced airglow excited by rfradiation. Radio Sci. 9, 1005-1019.

Kosch, M.J., Rietveld, M.T., Hagfors, T., Leyser, T.B., 2000. High-latitude HF-induced airglow displaced equatorwards of the pump beam. Geophys. Res. Lett. 27, 2817-2820.

Kosch, M.J., Rietveld, M.T., Kavanagh, A.J., Davis, C., Yeoman, T., Honary, F., Hagfors, T., 2002. High-latitude pump-induced optical emissions for frequencies close to the third electron gyro-harmonic. Geophys. Res. Lett. 29, 2112-2115.

Kosch, M.J., Pedersen, T., Hughes, J., Marshall, R., Gerken, E., Senior, A., Sentman, D., McCarrick, M., Djuth, F., 2005. Artificial optical emissions at HAARP for pump frequencies near the third and second gyroharmonic. Ann. Geophys. 23, 15851592.

Kosch, M.J., Pedersen, T., Rietveld, M.T., Gustavsson, B., Grach, S.M., Hagfors, T., 2007a. Artificial optical emissions in the thermosphere induced by powerful radio waves: an observational review. Adv. Space Res. 40 (3), 365-376.

Kosch, M.J., Pedersen, T., Mishin, E., Oyama, S., Hughes, J., Senior, A., Watkins, B., Bristow, B., 2007b. Coordinated optical and radar observations of ionospheric pumping for a frequency pass through the second electron gyro-harmonic at HAARP. J. Geophys. Res. 112, A06325, doi:10.1029/2006JA012146.

Kosch, M.J., Pedersen, T., Mishin, E., Starks, M., Gerken-Kendall, E., Sentman, D., Oyama, S., Watkins, B., 2007c. Temporal evolution of pump beam self-focusing at HAARP. J. Geophys. Res. 112, A08304, doi:10.1029/2007JA012264.

Leyser, T.B., Gustavsson, B., Brändström, B.U.E., Steen, Å., Honary, F., Rietveld, M.T. Aso, T., Ejiri, M., 2000. Simultaneous measurements of high-frequency pumpenhanced airglow and ionsopheric temperatures at auroral latitudes. Adv. Polar Upper Atmos. Res. 14, 1-11.

Leyser, T.B., Wong, A.Y., 2009. Powerful electromagnetic waves for active environmental research in geospace. Rev. Geophys. 47, RG1001, doi:10.1029/ 2007RG000235.

Mantas, G.P., Carlson, H.C., 1991. Re-examination of the O3P - > O1D excitation rate by thermal impact. Geophys. Res. Lett. 18, 159-162.

Mutiso, C.K., Hughes, J.M., Sivjee, G.G., Pedersen, T., Gustavsson, B., Kosch, M.J., 2008. Unreported optical emissions generated during ionospheric heating. Geophys. Res. Lett. 35, L14103, doi:10.1029/2008GL034563.

Oyama, S.-I., Watkins, B., Djuth, F.T., Kosch, M.J., Bernhardt, P., Heinselman, C.J., 2006. Persistent enhancement of the HF pump-induced plasma line measured with a UHF diagnostic radar at HAARP. J. Geophys. Res. 11, A06309, doi:10.1029/ 2005JA011363.

Pavlov, A.V., 1998a. New electron energy transfer rates for vibrational excitation of $\mathrm{N}_{2}$. Ann. Geophys. 16, 176-182.

Pavlov, A.V., 1998b. New electron energy transfer and cooling rates by excitation of $\mathrm{O}_{2}$. Ann. Geophys. 16, 1007-1013.

Pavlov, A.V., Berrington, K.A., 1999. Cooling rate of thermal electrons by electron impact excitation of fine structure levels of atomic oxygen. Ann. Geophys. 17, 919-924.

Pedersen, T.R., Carlson, H.C., 2001. First observations of HF heater-produced airglow at the high frequency active auroral research program facility: thermal excitation and spatial structuring. Radio Sci. 36, 1013-1026. 
Pedersen, T.R., McCarrick, M., Gerken, E., Selcher, C., Sentman, D., Carlson, H.C., Gurevich, A., 2003. Magnetic zenith enhancement of HF radio-induced airglow production at HAARP. Geophys. Res. Lett. 30 doi:10.1029/2002GL016096.

Pedersen, T., Esposito, R., Starks, M., McCarrick, M., 2008. Quantitative determination of HF radio-induced optical emission production efficiency at high latitudes. J. Geophys. Res. 113 doi:10.1029/2008JA013502.

Rees, M.H., Roble, R.G., 1975. Observations and theory of the formation of stable auroral red arcs. Rev. Geophys. Space Phys. 13, 201-242.

Rietveld, M.T., Isham, B., Kohl, H., La Hoz, C., Hagfors, T., 2000. Measurements of HF-enhanced plasma and ion lines at EISCAT with high altitude resolution. J. Geophys. Res., 7429-7439.

Rietveld, M.T., Kosch, M.J., Blagoveshchenskaya, N.R., Kornienko, V.A., Leyser, T.B. Yeoman, T.K., 2003. Ionospheric electron heating, optical emissions and striations induced by powerful HF radio waves at high latitudes: aspect angle dependence. J. Geophys. Res. 108, 1141, doi:10.1029/2002JA009543.

Rietveld, M.T., Kosch, M.J., Blagoveshchenskaya, N.F., Kornienko, V.A., Leyser, T.B. Yeoman, T.K., 2004. Correction to ionospheric electron heating, optical emissions, and striations induced by powerful $\mathrm{HF}$ radio waves at high latitudes: aspect angle dependence. J. Geophys. Res. 109, A04306, doi:10.1029/2004JA010460.

Robinson, T.R., Honary, F., Stocker, A.J., Jones, T.B., Stubbe, P., 1996. First EISCAT observations of the modification of F-region electron temperatures during RF heating at harmonics of the electron gyro frequency. J. Atmos. Terr. Phys. 58, 385-395.

Robinson, T.R., 1989. The heating of the high latitude ionosphere by high power radio waves. Phys. Rep. 179, 79-209.

Schunk, R.W., Nagy, A.F., 2004. Ionospheres: Physics, Plasma Physics, and Chemistry. Cambridge Univ. Press, Cambridge.

Sergienko, T., Gustavsson, B., Steen, A., Brändström, U., Rietveld, M., Leyser, T.B. Honary, F., 2000. Analysis of excitation of the $630.0 \mathrm{~nm}$ airglow during a heating experiment in Tromso on February 16, 1999. Phys. Chem. Earth (B) 25, 531-535.

Stubbe, P., Varnum, W.S., 1972. Electron energy transfer rates in the ionosphere. Planet. Space Sci. 20, 1121-1126. 